\title{
Contribution of Diffusion-Weighted Magnetic Resonance Imaging in the Follow-Up of Uncomplicated Acute Appendicitis: Initial Results Oguzhan Ozdemir,,$^{, 2}$ Nurgul Orhan Metin, ${ }^{1}$ Yavuz Metin, ${ }^{1}$ Suleyman Kalcan, ${ }^{2}$ and Ali Kupeli ${ }^{3}$
}

${ }^{1}$ Department of Radiology, Faculty of Medicine, Recep Tayyip Erdogan University, Rize, Turkey

${ }^{2}$ Department of General Surgery, Faculty of Medicine, Recep Tayyip Erdogan University, Rize, Turkey

${ }^{3}$ Department of Radiology, Mus State Hospital, Mus, Turkey

"Corresponding author: Oguzhan Ozdemir, Recep Tayyip Erdogan University, Faculty of Medicine, Department of Radiology, Rize, Turkey. Tel: +49-5335182641, Fax: +46-642345324, E-mail: droguzrad@hotmail.com

Received 2017 January 17; Revised 2017 June 24; Accepted 2017 October 09

\begin{abstract}
Background: Several studies have shown that the majority of patients with simple appendicitis can be treated effectively and safely with antibiotics, avoiding unnecessary operation.

Objectives: The objective of this study was to assess the role of diffusion-weighted imaging (DWI) in the follow-up of conservative treatment of simple appendicitis.

Patients and Methods: Twenty-eight patients (mean age, $35 \pm$ 14.6; age range, 18 - 72; M/F: 16/12) with simple appendicitis were enrolled in this prospective study. After the primary diagnosis by computed tomography (CT) at presentation, they underwent an initial DWI scan, and intravenous antibiotic therapy was started for all patients by the decision of the attending surgeon. A total of three control DWI scans were planned during the follow-up period for each patient. CT and DWI images were evaluated by two radiologists in consensus. B values 0,500 and $1000 \mathrm{~s} / \mathrm{mm}^{2}$ were used, and DWI images were analyzed both qualitatively and quantitatively. During follow-up, changes in the diameter of appendices and laboratory parameters were correlated with apparent diffusion coefficient (ADC) values.

Results: We found statistically significant correlation between ADC values, maximum appendix diameter and laboratory parameters. During follow-up period four surgical procedures were performed for reccurent appendicitis.

Conclusion: DWI may be used with a significant success for follow-up evaluation of patients with simple appendicitis. As a monitoring imaging method, DWI may also aid in determining the recurrences of appendicitis.
\end{abstract}

Keywords: Acute Appendicitis, Computed Tomography, Diffusion-Weighted Imaging

\section{Background}

Acute appendicitis (AA) is a common surgical emergency, with an estimated lifetime risk of $7 \%-8 \%$. Appendicitis is divided into two categories: (a) uncomplicated (simple) appendicitis and (b) complicated appendicitis. Surgery is accepted as the best treatment option for AA. Alternatively, it has been reported that simple appendicitis (SA) is a mild inflammation that may resolve either spontaneously or with antibiotics (1). Although most patients tolerate surgery, risks of postoperative complications have been reported to occur in up to $23 \%$ of patients $(2,3)$. Additionally, some studies have shown that $3 \%$ of patients undergoing an appendectomy with or without laparoscopy were readmitted for mechanical obstruction, which is also a postoperative complication $(4,5)$. Several studies have shown that the majority of patients with simple appendicitis can be treated effectively and safely with antibiotics, thereby avoiding unnecessary surgery (6).

Prompt and early diagnosis is critically important as delay in the diagnosis may result in several complications that may increase morbidity, and even mortality. Pre-operative imaging is reported to decrease falsepositive appendectomy rates; therefore, imaging is commonly utilised to assess AA. Ultrasonography(US) and computed tomography (CT) are two types of workhorse imaging modalities that are used for the primary evaluation of AA patients, and CT is used more often than US (7-11). Of these two modalities, CT has been reported to be more sensitive than US for primary diagnosis and for identifying potential complications $(12,13)$. Despite these advantages, the use of CT is limited for patients in the paediatric age group and pregnant patients in whom US is more commonly being employed $(14,15)$. In most patients, the CT features of AA include enlargement of the appendix (over $6 \mathrm{~mm}$ ), wall thickening with enhancement, periappendiceal fat strand- 
ing, the presence of an appendicolith and, in some cases, focal thickening of the terminal ileum or cecum. With $\mathrm{CT}$, traditionally a cut-off value $>6 \mathrm{~mm}$ has been used to diagnose AA. However, several studies have demonstrated that a cut-off value of $6 \mathrm{~mm}$ is too low on cross-sectional imaging; instead, an 8 - $9 \mathrm{~mm}$ cut-off value has been suggested $(12,13,16)$. In simple appendicitis, an appendix appears as an enlarged, fluid-filled tubular structure with an intact wall and slight periappendiceal fat stranding or fluid. Complicated appendicitis is indicated by the presence of a focal defect in an enhanced appendiceal wall, periappendiceal fluid collection, pericecal phlegmon, an extraluminal appendicolith, or air near the appendix. A pericecal phlegmon or abscess strongly suggests a perforated appendicitis. However, these findings are nonspecific, and they may be seen with other disease processes involving the cecum and terminal ileum (17). In a study using a scoring system that combined clinical and CT imaging features, 95\% of patients with SA were correctly diagnosed (18). It has been reported that the importance of accurate AA diagnosis and the differential diagnosis of complicated and SA have led to the widespread use of diagnostic imaging (17).

Although magnetic resonance imaging (MRI) is not often used to diagnose AA, several studies have reported the benefits of utilizing MRI techniques in an emergency setting $(19,20)$. Diffusion-weighted magnetic resonance imaging (DWI) of the abdomen and pelvis has been increasingly used since the 1990s, with the introduction of stronger diffusion gradients, faster imaging sequences and improvements in MRI hardware. DWI can be performed in a very short time without using any intravenous (IV) contrast media, thereby allowing fast, qualitative assessment (21-24). DWI is reported to have a sensitivity of $99 \%$ and a specificity of $97 \%$ in the diagnosis of AA in adults using a b value of $500 \mathrm{~s} / \mathrm{mm}^{2}$ (25). In a DWI study on perforated versus nonperforated appendicitis, the apparent diffusion coefficient (ADC) values were found to be lower in perorated than in nonperforated appendicitis (26).

\section{Objectives}

This prospective study aims to assess the value of using DWI in the follow-up of antibiotic-treated SA in order to: 1) evaluate the antibiotic response of the infectiousinflammatory process of AA, 2) correlate the ADC values with laboratory parameters and 3 ) conduct imaging to detect AA relapses.

\section{Patients and Methods}

\subsection{Patient Selection and Inclusion Criteria}

For this prospectively designed study, from September 2014 to October 2016, we used CT to scan 328 patients presenting with symptoms that were suggestive of AA. The CT findings of 186 of those patients showed simple SA. The type of therapy was based on the decision of the surgical team. The study design was constructed by radiologists and surgeons. Patients with the following features of appendicitis were included in the study: 1) those with SA without appendicoliths, 2) those with stable clinical status, without a serious comorbidity and 3) those only accepting antibiotic therapy, knowing the potential benefits and other outcomes of this type of therapy. The general surgeons asked all the patients meeting these criteria to undergo a conservative therapeutic approach. Only 31 of those patients received medical treatment, based on the decision of the attending surgeon in charge, with IV antibiotics and fluid resuscitation; none of those patients underwent emergency surgery at presentation. Instead, they underwent DWI after a CT scan. Since the appendices of three of the 31 patients could not be visualized via DWI, only 28 patients (mean age, $35 \pm 14.6$; age range, 18 - 72; $\mathrm{M} / \mathrm{F}: 16 / 12$ ) were included in the present study. These three patients were put on conservative therapy without screening by DWI. A total of 297 patients underwent immediate surgery. Pregnant women, unstable patients, patients under the age of 18 and those with appendicoliths were not included in the study. This study was approved by the Medical ethics committee of our university hospital. Written consent was obtained from each patient after a discussion regarding the potential benefits and disadvantages of the procedure, and additional written consent was obtained before the MRI in all 28 patients. During the follow-up, and if symptoms of recurrent appendicitis developed, only DWI without IV contrast media was performed. Age, gender, clinical characteristics and laboratory findings of the patients were documented. The patient characteristics at presentation are shown in Table 1 . After the first DWI was performed at presentation, a total of three control DWI scans were planned during the follow-up period. Control DWI studies were performed at the end of the first, second and eighth weeks in the early follow-up period. Two months after the index study, the patients were called in for a clinical visit every three months (Table 2).

\subsection{Imaging Protocols}

CT was performed with a 128-row CT (Somatom Definition AS, Siemens, Erlangen, Germany). The following CT protocol was used: $120 \mathrm{kVp}$, tube current of 150 - $165 \mathrm{mAs}$, maximum $2.5 \mathrm{~mm}$ collimation, a $3 \mathrm{~mm}$ slice thickness and 
Table 1. Patient Characteristics at Presentation $(\mathrm{N}=28)$

\begin{tabular}{|lcc|}
\hline Variables & No. & Percentage, $\%$ \\
\hline Gender & 16 & 57.1 \\
\hline Male & 12 & 42.8 \\
\hline Female & & \\
\hline Pain presentation of patients & 8 & 28.5 \\
\hline Generalized abdominal pain & 12 & 42.8 \\
\hline Right lower quadrant pain & 8 & 28.5 \\
\hline Generalized pelvic pain & & \\
\hline Fever & 3 & 10.7 \\
\hline+ & 25 & 89.2 \\
\hline - & & \\
\hline
\end{tabular}

a 0.5 seconds rotation time, portal venous phase images (scanning delay, 60 - 70 seconds) with a total of $100 \mathrm{~mL}$ nonionic contrast agent containing an iodine concentration of $300 \mathrm{mg} / \mathrm{mL}$ injected via a power injector at a rate of $4 \mathrm{~mL} / \mathrm{s}$ $\mathrm{r}$ (Medrad ${ }^{\circledR}, \mathrm{CT}$ injection system, Bayer). The scanning area ranged from the diaphragm to the pelvis, with the patient placed in the supine position. None of the patients were given oral contrast.

In the 28 patients that underwent DWI, the DWI studies were performed with a 1.5-T MR scanner (Magnetom ${ }^{\circledR}$ Aera; Siemens, Erlangen, Germany). The following DWI protocol was used: axial diffusion-weighted single-shot echoplanar sequence (EPI) with fat suppression, without breath holding (repetition time (TR), 7500; time to echo (TE) 62 - 80 ms; matrix, $192 \times 192$; slice thickness, $5 \mathrm{~mm}$; gap, $2 \mathrm{~mm}$; field of view (FOV), $400 \mathrm{~mm}$; parallel acquisition technique (PAT) factor 2; acquisition time, 3 minutes; b values 0, 500, and $1000 \mathrm{~s} / \mathrm{mm}^{2}$ ). As in the CT, each patient's abdomen was scanned from the diaphragm to the pelvis.

\subsection{Image Analysis}

Two abdominal radiologists, with 10 and 5 years of experience, interpreted the CT scans of all patients. Initial and follow-up MRI studies of the 28 patients enrolled in this study were also assessed by the same radiologists. All interpretations were made via consensus. When the decisions were discordant, a collaborative discussion occurred, and final consensus was reached. The radiologists were aware of the clinical and laboratory findings. For all CT studies $(n=28)$ performed at the time of initial presentation, sagittal and coronal reformatted images were also created in addition to standard axial images.

At the time of initial presentation, the following CT features, were searched for in all patients with clinical and laboratory findings that were suggestive of AA: the diameter of the appendix, the integrity of the appendiceal wall, the presence of appendicolith, surrounding fat stranding and the presence of abdominal fluid or air or abscess formation. AA was considered when the appendiceal diameter exceeded $8 \mathrm{~mm}$ with wall thickening and at least one of the following was present: abnormal contrast enhancement of the appendiceal wall and periappendiceal fat stranding or fluid. The CT criteria for SA were: an enlarged appendix without a defect of the appendiceal wall and mild periappendiceal fat stranding or fluid. For a follow-up diagnosis of SA, we also excluded patients with appendicoliths. A diagnosis of complicated appendicitis was considered when a defect of the appendiceal wall was found in the presence of prominent periappendiceal fat stranding, fluid and any extraluminal air, phlegmon or abscess.

For DWI, the appendixes were qualitatively assessed in images acquired at b1000 values. DWI findings suggesting a diagnosis of AA were an enlarged hyperintense appendix, periappendiceal fat stranding and surrounding fluid. At presentation, after measuring the appendiceal diameter on CT, a primary measurement was done on the first DWI and used as an index value for the follow-up DWIs. During follow-up, we also measured the appendiceal diameter on b 1000 images. The b 0 and b 500 images were used to obtain anatomical details. ADC maps were also evaluated for all DWI studies to obtain quantitative diffusion restriction data. The decision as to whether the diagnosis was simple or complicated appendicitis was made using CT results rather than DWI results.

In all 28 patients, the initial CT and the initial and follow-up DWI datasets were evaluated at an independent workstation (Syngo.via, Siemens) for CT post-processing and ADC map analysis. The CT images were evaluated before the DWI images were analyzed. The initial CT scan parameters mentioned above were recorded, and then the corresponding lesions were again evaluated, this time, using DWI and ADC maps. In the follow-up DWI studies, we mainly evaluated the size (by measuring the longest diameter on the $\mathrm{b} 1000$ images) and the evolution of the diffusion restriction using ADC maps. The ADC values were measured by placing a circular region of interest (ROI) on the inflamed appendix covering both the lumen and the wall. The size of the ROI was kept as large as possible covering the most hypointense parts of the appendix on the ADC maps. The mean of three separate ROI measurements was accepted as the ADC value for each patient. The same 
Table 2. Changes of Parameters at Presentation and Follow-Up Period

\begin{tabular}{|c|c|c|c|c|}
\hline Variables & Minimum & Maximum & Mean & Std. Deviation \\
\hline \multicolumn{5}{|c|}{ ADC, $\times 10^{-3} \mathrm{~mm}^{2} / \mathrm{s}$} \\
\hline First & 0.99 & 1.53 & 1.30 & 0.14 \\
\hline Second & 1.49 & 1.86 & 1.65 & 0.11 \\
\hline Third & 1.77 & 2.14 & 1.90 & 0.07 \\
\hline Fourth & 1.94 & 2.22 & 2.04 & 0.08 \\
\hline \multicolumn{5}{|l|}{ CRP, mg/L } \\
\hline First & 5.3 & 9.3 & 7.2 & 1.1 \\
\hline Second & 2.8 & 5.5 & 4.4 & 0.6 \\
\hline Third & 1.4 & 4.3 & 2.9 & 0.7 \\
\hline Fourth & 1.6 & 3.5 & 2.4 & 0.5 \\
\hline \multicolumn{5}{|l|}{ DIA, mm } \\
\hline First & 8 & 11.7 & 9.4 & 0.9 \\
\hline Second & 5.9 & 9 & 7.3 & 0.7 \\
\hline Third & 5.1 & 6.8 & 6.1 & 0.4 \\
\hline Fourth & 5 & 6.5 & 5.7 & 0.4 \\
\hline \multicolumn{5}{|l|}{ WBC, per mL } \\
\hline First & 9700 & 23690 & 15876 & 4044 \\
\hline Second & 7320 & 12000 & 8611 & 2170 \\
\hline Third & 5030 & 9500 & 7318 & 1120 \\
\hline Fourth & 5070 & 8940 & 6921 & 1003 \\
\hline
\end{tabular}

Abbreviations: ADC, apparent diffusion coefficient; CRP, C-reactive protein; DIA, diameter; WBC, white blood cell count.

measurements were repeated on the follow-up MRI studies, placing the ROIs as much as possible on similar parts of the appendixes taking the previously measured regions into account. For each follow-up DWI scan, the radiologists were aware of the previous imaging findings and the clinical and laboratory findings.

\subsection{Clinical Analysis and Type of Conservative Therapy}

At presentation, the presence of abdominal pain and fever, and the levels of C-reactive protein (CRP) and white blood cell (WBC) counts were noted. The same parameters were re-assessed in the follow-up visits. All patients $(n=28)$ were hemodynamically stable, and none were critically ill at the time of initial presentation. Thus, the attending surgeon in charge concluded that conservative therapy was the appropriate treatment. All 28 patients were initially hospitalized and administered IV ceftriaxone ( $1-2 \mathrm{~g} /$ day in single daily dose) combined with metronidazole $(500 \mathrm{mg}$,
3 times per day). The in-hospital IV antibiotic treatment was continued for three days for each patient. Afterwards, oral therapy with the same doses was continued for an extra seven days to complete the 10-day course of treatment. To rule out any underlying malignancy, patients over the age of 40 also planned to have a colonoscopy four weeks after the index episode.

\subsection{Statistical Analysis}

All the data were analyzed using Statistical Package for Social Sciences software (SPSS 13.0 Statistical Software, SPSS Inc., Chicago, IL, USA). The medians and ranges for age, CRP levels, WBC, appendiceal diameter and ADC values were calculated. The Kolmogorov-Smirnov test was used to show deviation from normal distribution. Spearman's correlation analysis was used to analyze the correlation between ADC values and serum CRP levels, WBC and appendiceal diameter. Within each treatment period, changes in 
serum CRP levels, ADC values, WBC, and appendiceal diameter were evaluated using repeated measures ANOVA with Greenhouse-Geisser correction. Post hoc comparisons between the first time point and each additional time point were performed using Student's t-test followed by Bonferroni adjustment of all probability values on the basis of the number of comparisons made in each period. The paired T-test was used to compare the mean values of appendiceal diameters measured on CT and DWI images at presentation. The Mann-Whitney U test was used to compare ADC values between patients with appendicitis relapse and those who did not relapse. A p value of less than 0.05 was considered as statistically significant.

\section{Results}

The patient flow diagram is shown in Figure 1. At presentation, all patients were hospitalized; the mean hospital stay was 2.92 ( \pm 0.97; range, 2 - 5 days) days. No statistically significant difference was found between the means of appendiceal diameters measured on CT (9.2 mm \pm 0.7$)$ and DWI (9.4 $\mathrm{mm} \pm 0.9)$ at presentation $(\mathrm{P}=0.785)$.

\subsection{Clinical Outcomes}

The mean follow-up time was $12( \pm 4)$ months, ranging from 6 to 20 months. During follow-up, four patients came to the emergency department with acute right lower quadrant pain. They were imaged with DWI and diagnosed with recurrent AA. All four patients underwent an emergency appendectomy within a few hours, based on the attending surgeon's decision. The DWI for those patients showed thickened hyperintense appendixes with restricted diffusion on ADC maps. The remaining patients $(n=24)$ did not develop any relapse or complications; and, when this article was written, that was still the case. The patients were warned about the potential risk of developing a relapse in the future, and they were informed that they should go to the emergency department as soon as possible, should that occur.

Eleven of the patients in our study group were over the age of 40, and they underwent a colonoscopy the fourth week after the index presentation. None of those patients demonstrated any evidence of malignancy or inflammatory bowel disease on their elective fourth week colonoscopy exams.

\subsection{The Evolution of the Parameters During Follow-Up}

CRP levels, WBC, ADC values, and appendiceal diameter were the main parameters that were recorded during the follow-up period. The interval evolution of these parameters both at the initial presentation and on the follow-up scans was a slope showing a decrease in serum CRP levels, WBC values and appendix diameter, while ADC values progressively decreased. Changes in these parameters demonstrated a significant inverse correlation with ADC values ( $P$ $<0.001$ ). The ANOVA test results indicated a significant change in the appendix diameter, ADC value, serum CRP value and WBC value of the 28 patients during follow-up. For each parameter, the mean difference at presentation and follow-up was found to be statistically significant $(\mathrm{P}<$ 0.01). Serum CRP levels, WBC values and appendiceal diameters showed a strong inverse significant correlation with ADC values (CRP, $\mathrm{r}=-0.894, \mathrm{P}<0.001$; $\mathrm{WBC}, \mathrm{r}=-0.746, \mathrm{P}<$ 0.001; appendiceal diameter, $\mathrm{r}=-0.873, \mathrm{P}<0.001$ ) (Figures 2).

Analysis of the DWI images assessed in patients with relapses was sufficient to detect the inflamed appendixes against a suppressed background signal; the hyperintense signal on the DWI and the corresponding diffusion restriction on the ADC maps were easily discernible for all patients. We did not find any significant difference in the change of the means of ADC values between patients with relapses $(n=4)$ and the rest of the patients $(n=24)$ during the follow-up period in which DWIs were conducted $(\mathrm{P}=$ $0.681)$.

\section{Discussion}

Computed tomography is the type of imaging modality that is most commonly used to diagnose appendicitis and its complications. The type of appendicitis (complicated or simple) and the associated anatomic relationships are well depicted with CT, which also helps determine the appropriate treatment approach (27-29). In the present study, the CT images clearly showed SA in all 28 patients based on the CT criteria mentioned above.

In the surgical literature, appendectomy has been the gold-standard treatment for AA. However, non-surgical treatment is the current trend for treating simple appendicitis. This approach aims to reduce healthcare costs and avoid the potential complications related to surgery. Recently, new studies on adult and pediatric age groups have been encouraging (30). The Appendicitis Acuta (APPAC) study is a recently published study of a large adult age group that reported on antibiotic therapy for simple appendicitis (6). Their findings showed that most patients with CT-proven SA who received antibiotic therapy during the first one-year follow-up did not require an appendectomy, and those who underwent surgery did not have significant complications. In our study, the percentage of recurrent appendicitis was lower than the percentage reported in the APPAC study (27.3\%), while in the present study only four patients (14.2\%) developed acute relapse of 


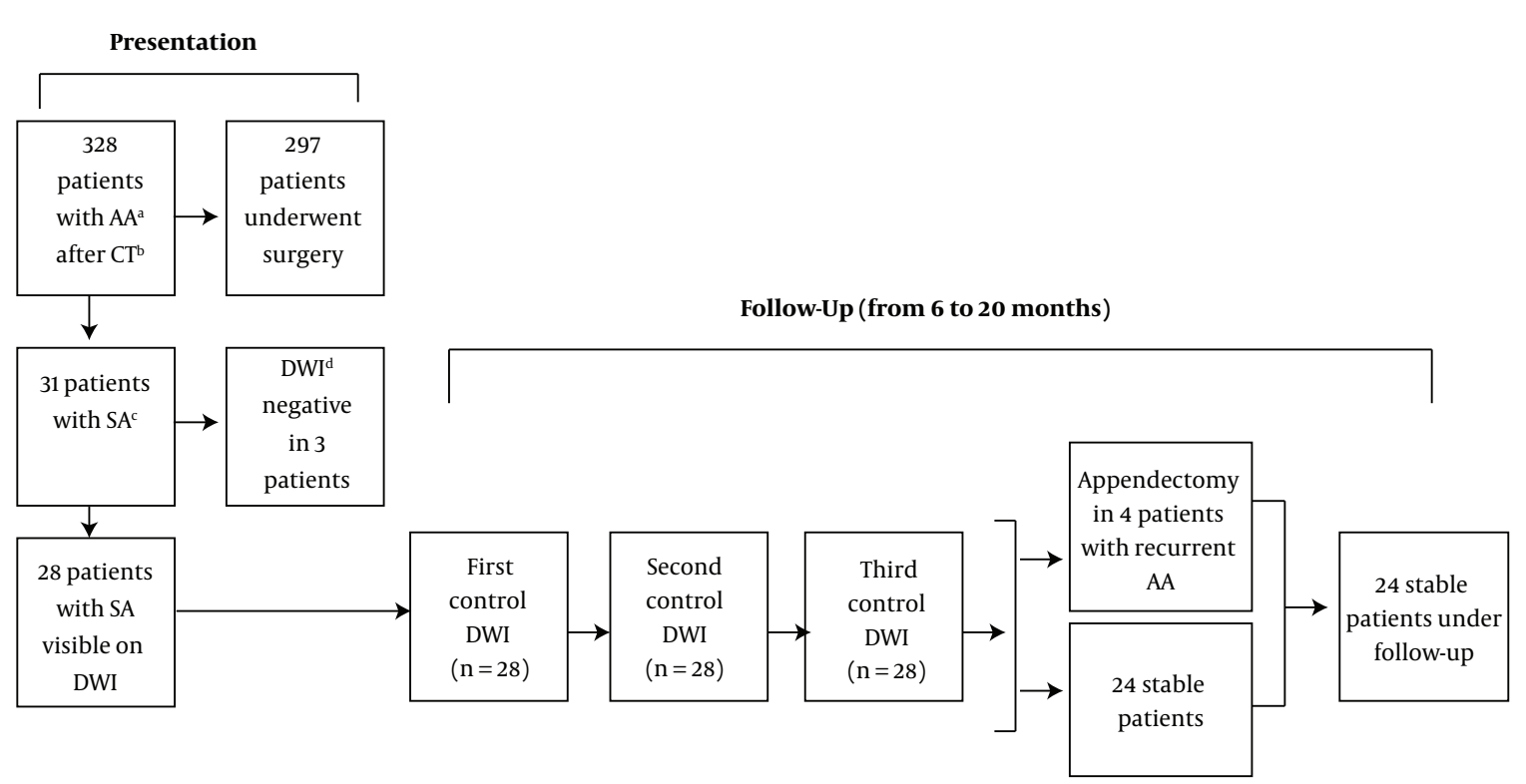

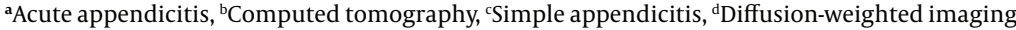

Figure 1. Patient flow diagram

appendicitis, and they underwent an emergency appendectomy within a few hours of the relapse without any complications. The higher rate of acute relapse in the APPAC study might be due to the inclusion of patients with appendicoliths. In a recently published pediatric study, the recurrence rate was found to be $5.3 \%$ for patients with appendicoliths and $0 \%$ for patients without appendicoliths (31).

In another study (32) reported that appendicoliths were significantly associated with a greater risk for complicated appendicitis. They concluded that appendicoliths were associated with antibiotic therapy failure. This finding also emphasizes the significance of imaging to rule out appendicoliths. Knowing this fact, we excluded appendicitis associated with appendicoliths even if the patient was diagnosed with SA. Diffuse peritonitis and intraabdominal abscess are the most severe complications of appendicitis (6). It has been suggested that in CT-proven SA, a delay in surgery due to antibiotic therapy does not increase the risk of complications (6). To date, none of the patients in our study have faced any complications during follow-up.

In the APPAC study, the incidence of tumors was found to be $1.5 \%$, which is in concordance with the literature (6). That study stated that imaging facilitated the detection of incidental tumors (6). In general, colonoscopy is recommended in patients over the age of 40 to rule out se- rious conditions when conservative management is utilized. In our study, 11 patients over the age of 40 underwent colonoscopy during the fourth week follow-up period; pathology was not found in any of these patients. However, during follow-up of SA, even with a colonoscopy, diagnosis of an underlying tumor or inflammatory bowel disease may still be problematic, and imaging may be required. It has been reported that in conditions in which therapeutic alternatives to surgery are favored, patient satisfaction might be also problematic during follow-up (31). When considering these facts and patient satisfaction during follow-up, imaging may become critical. In this respect, imaging may play a key role. The unique features of DWI (free of ionizing radiation and no need for a contrast agent) may make it an alternative imaging method during follow-up when conservative management of simple appendicitis is ordered.

In modern medical practice, imaging is widely used to diagnose AA; with the widespread use of modern imaging technology, false positive appendectomy rates have significantly decreased $(2,13)$. With the increasing use of imaging, an alternative diagnosis that might mimic AA may be made with high success rates. In patients who receive conservative medical treatment for SA rather than undergoing immediate surgery, imaging may play a fundamental role in the follow-up. In reviewing the literature, we have not found any well-studied standard imaging method for 
A

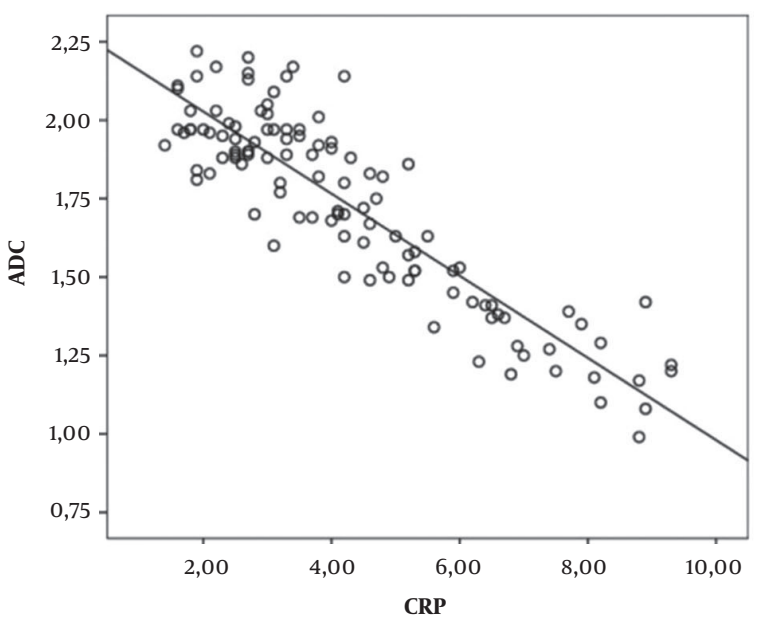

B

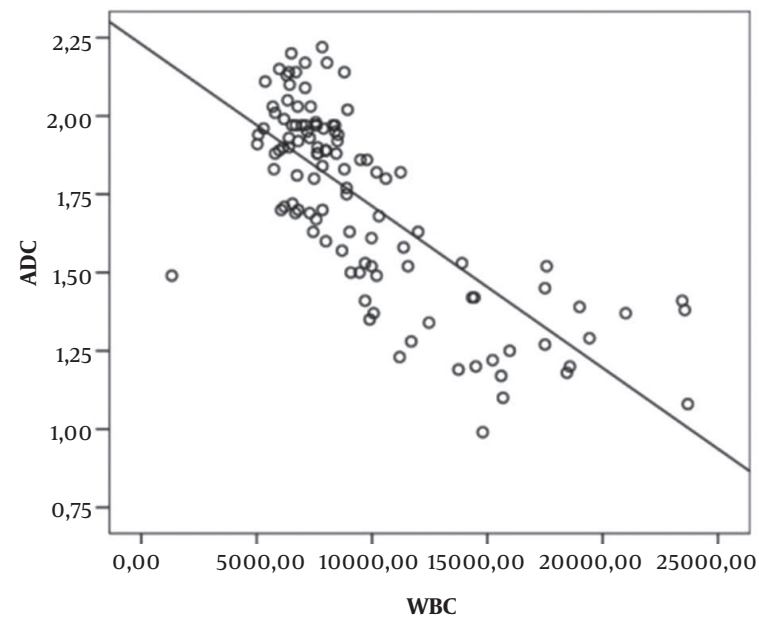

C

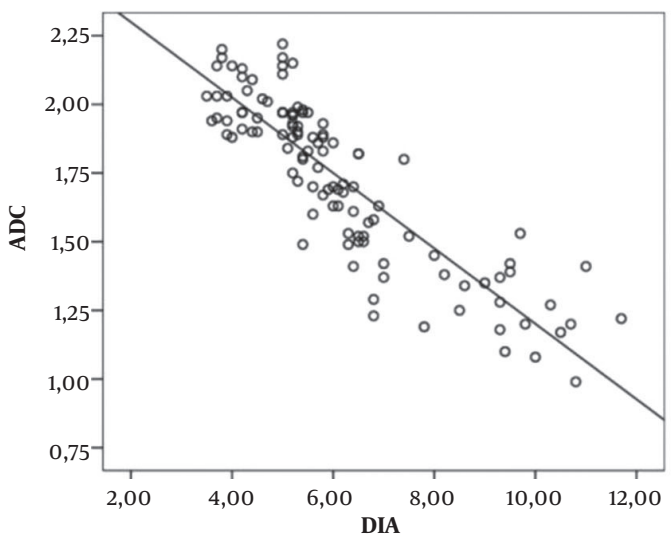

Figure 2. Graphs show the correlation between A, C reactive protein (CRP) and apparent diffusion coefficient (ADC) values; B, White blood cell (WBC) and ADC values and C, Appendiceal diameter and ADC values.

follow-up for antibiotic-treated simple appendicitis. Conventionally, CT and US are the most commonly used modalities for the diagnosis of AA and its complications. In spite of this, imaging during follow-up is still controversial. This is critical when considering the risks of alternative diagnoses (e.g. tumors or inflammatory bowel disease) and the risk of recurrence of appendicitis that is reported to be $25 \%$ - 30\% during first-year follow-up (1). An initial CT scan has been reported to improve patient care and reduce costs, regardless of whether the patient receives antibiotic therapy or undergoes surgery (6). However, there are disadvantages to both US and CT. US is highly operatordependent, and it may be less sensitive, especially in patients with large body habitus. Bowel distension is another limiting factor in US, obscuring the underlying posteriorly located pathologies. CT is an excellent tool for abdominal imaging; it can be quickly performed in a matter of seconds and it is also widely available. However, the main disadvantage of using repeat CT scans is the overall accumulation of radiation, especially in the pediatric age group and young adults. The cumulative IV contrast load may also be problematic in patients who are allergic to contrast media or who have low renal reserves. In such cases, a low-dose CT scan may be an option; however, the use of cumulative radiation and contrast media will be still problematic with repeated scans.

Magnetic resonance imaging may be a perfect method in these groups of patients, with its lack of radiation and excellent soft tissue resolution. There are also disadvantages to using MRI, including longer imaging time, lack of 

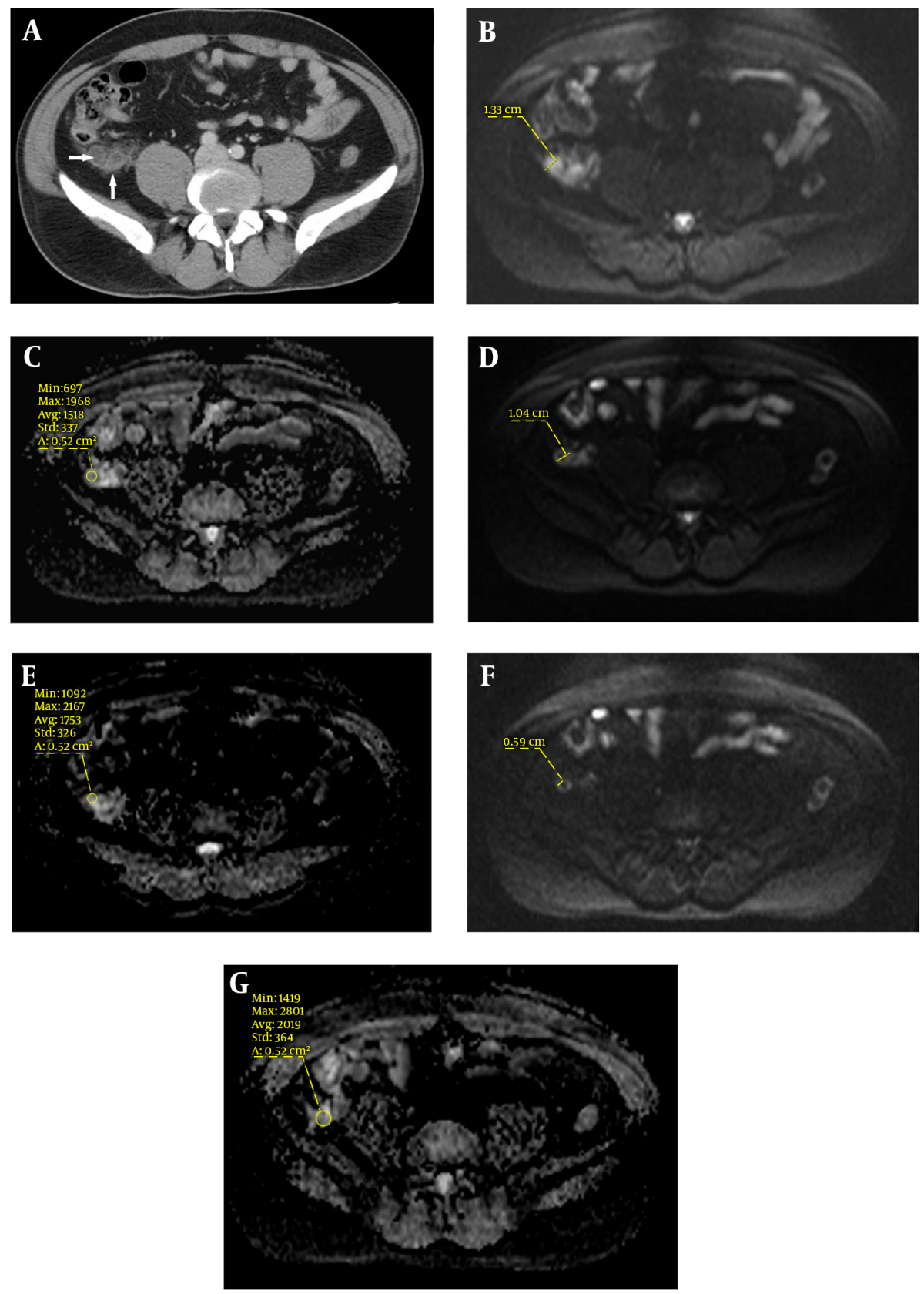

Figure 3. A 26-year-old male with right lower quadrant pain for a day. Clinical and laboratory findings suggested acute appendicitis. Antibiotic therapy was started for the patient. A, Contrast-enhanced axial CT reveals an enlarged appendix with some periappendiceal fat stranding compatible with simple appendicitis (arrows). B, Diffusion weighted imaging (DWI) at presentation, shows the inflamed appendix with surrounding hyperintense edema. C, Apparent diffusion coefficient (ADC) map at presentation shows diffusion restriction. D, Control DWI at the end of first week depicts regression of inflammation with decrease of appendiceal diameter and surrounding edema. E, ADC map shows an increase of ADC value. F, Control DWI at the end of fourth week shows that the appendiceal inflammation and surrounding edema has completely decayed. G, ADC map reveals the progressive increase of ADC value. 
local expertize and other logistical challenges. The use of DWI as the only imaging sequence for follow-up of these patients may significantly help in overcoming some of the limitations of MRI. DWI is a very fast imaging sequence, which also helps patients avoid the burden of cumulative radiation and contrast media. DWI provides qualitative and quantitative analysis at the cellular level. While it is considered to be an effective type of functional imaging (33), some morphological information may also be acquired. Conventionally, DWI is most commonly used in neuroimaging applications; however, with the recent advances in MRI technology, body applications are becoming more common. Restricted diffusion is commonly observed if high cellularity is found within the lesion (e.g. tumors, abscesses, fibrosis and cytotoxic edema). DWI is sensitive to the micro-environmental changes in tumors at the molecular level that result from treatment; thus, it may predict tumor response to treatment (34). DWI may also be used as an imaging tool to monitor infectiousinflammatory processes to evaluate the evolution of disease during a conservative treatment period, as we tried to determine whether DWI could be used to monitor the evolution of simple appendicitis with antibiotic therapy.

In the present study, we evaluated DWI as an imaging tool to assess changes in inflamed appendices, qualitatively and quantitatively, during follow-up (Figure 3). We evaluated the relationship between ADC values of appendixes, and we correlated the imaging data with the laboratory results (CRP and WBC). We also used morphological information provided by DWI to assess the dimensions of the appendixes. We found a statistically significant correlation between ADC values and laboratory parameters. We observed that, as ADC values increased (which suggests decreased cellularity within the appendix) serum inflammatory markers (i.e., CRP and WBC) normalized and the appendix diameter decreased (Figure 2). A statistically significant correlation was found between the increase in ADC values and the decrease in the appendix diameter, WBC counts and serum CRP levels $(\mathrm{P}<0.001)$. These findings may show that utilization of DWI may allow practitioners to evaluate the clinical response to conservative therapy by providing qualitative and quantitative data about appendixes. Knowing the primary infectious-inflammatory focus (in this case, SA during the early period of follow-up of conservative therapy might support the use of DWI as an effective monitoring tool instead of analyzing serum inflammatory markers. However, this needs to be investigated with a larger patient population with detailed data analysis.

During follow-up, four patients developed acute right lower quadrant pain and were found to have recurrent appendicitis visualised by DWI. The DWI findings were com- patible with the clinical findings and the laboratory results. All four patients underwent emergency surgery, and the pathological specimens revealed SA without perforation. Knowing that there was no statistically significant difference in the $\mathrm{ADC}$ values of the relapse and non-relapse patients during the early periods of follow-up, DWI did not help in anticipating recurrent appendicitis. However, this must be further studied with larger patient groups.

In the present study, the length of the primary hospitalization at presentation was similar to what was reported in the APPAC study (mean 3 days) with a mean of 2.92 ( \pm 097 ) days. In the APPAC study (6), the length of hospital stay was statistically significantly shorter in the surgical group than in the antibiotic-treated group $(\mathrm{P}<0.001)$. Unfortunately, our study did not have a surgical control group, so we were unable to compare that variable with the hospital stay length of surgical patients reported in the APPAC study.

Our study had several limitations. We only included 28 patients in this study, and this limited number may decrease the accuracy of our findings. For a better statistical assessment, larger patient groups with longer followup periods should be enrolled into future studies based on the information provided in this study. Our study did not have a control group without any screening protocol to compare the DWI results of the patients during the followup period to address the potential benefits of DWI. Another important limitation of this study was that we did not assess inter-observer variability. Moreover, we did not include other MRI sequences that could provide morphological information; as such, the lesion borders could have been more effectively identified, especially with the use of IV contrast media. However, the inclusion of other MRI sequences would go against the aim of this study, as we tried to implement the fastest MRI method without the use of IV contrast media. Finally, in the present study, the concept of performing repeated DWIs contradicts the efforts of conservative therapy for simple appendicitis, which aims to reduce healthcare costs.

In conclusion, we think that DWI would be highly successful for the follow-up of patients with SA who received antibiotic therapy instead of undergoing emergency surgery. Although medical history, physical examination findings and serum inflammatory markers are critically important for the diagnosis and follow-up of AA, DWI may be highly effective during early periods of conservative therapy. Furthermore, the lack of radiation in MRI may be especially useful in the follow-up of pregnant patients and pediatric patients. Although not present in our study patients, DWI may also help in diagnosing alternative conditions mimicking AA such as cecal cancer or inflammatory bowel disease, during follow-up. Finally, as DWI does 
not require IV contrast media, its use may be critical in patients with renal impairment.

\section{Footnotes}

Authors' Contributions: None declared.

Financial Disclosure: All authors declared that they had no conflict of interest.

Funding/Support: No funding or sponsorship was received for this study.

\section{References}

1. Bhangu A, Soreide K, Di Saverio S, Assarsson JH, Drake FT. Acute appendicitis: modern understanding of pathogenesis, diagnosis, and management. Lancet. 2015;386(10000):1278-87. doi: 10.1016/S01406736(15)00275-5. [PubMed: 26460662].

2. Drake FT, Florence MG, Johnson MG, Jurkovich GJ, Kwon S, Schmidt Z, et al. Progress in the diagnosis of appendicitis: a report from Washington State's Surgical Care and Outcomes Assessment Program. Ann Surg. 2012;256(4):586-94. doi: 10.1097/SLA.ob013e31826a9602. [PubMed: 22964731].

3. Humes DJ, Simpson J. Acute appendicitis. BMJ. 2006;333(7567):530-4. doi: 10.1136/bmj.38940.664363.AE. [PubMed: 16960208].

4. Puylaert JB. Ultrasonography of the acute abdomen: gastrointestinal conditions. Radiol Clin North Am. 2003;41(6):1227-42. vii. doi: 10.1016/S0033-8389(03)00120-9. [PubMed: 14661668].

5. Doria AS, Moineddin R, Kellenberger CJ, Epelman M, Beyene J, Schuh $S$, et al. US or CT for Diagnosis of Appendicitis in Children and Adults? A Meta-Analysis. Radiology. 2006;241(1):83-94. doi: 10.1148/radiol.2411050913. [PubMed: 16928974].

6. Levine CD, Aizenstein O, Wachsberg RH. Pitfalls in the CT diagnosis of appendicitis. BrJRadiol.2004;77(921):792-9. doi:10.1259/bjr/95663370. [PubMed: 15447972].

7. Kim K, Kim YH, Kim SY, Kim S, Lee YJ, Kim KP, et al. Low-dose abdominal CT for evaluating suspected appendicitis. $N$ Engl $J$ Med. 2012;366(17):1596-605. doi: 10.1056/NEJMoa1110734. [PubMed: 22533576].

8. Ives EP, Sung S, McCue P, Durrani H, Halpern EJ. Independent predictors of acute appendicitis on CT with pathologic correlation. Acad Radiol. 2008;15(8):996-1003. doi: 10.1016/j.acra.2008.02.009. [PubMed: 18620120].

9. Kim HC, Yang DM, Jin W, Park SJ. Added diagnostic value of multiplanar reformation of multidetector CT data in patients with suspected appendicitis. Radiographics. 2008;28(2):393-405. discussion 405-6. doi: 10.1148/rg.282075039. [PubMed: 18349447].

10. Atema JJ, van Rossem CC, Leeuwenburgh MM, Stoker J, Boermeester MA. Scoring system to distinguish uncomplicated from complicated acute appendicitis. BrJSurg. 2015;102(8):979-90. doi:10.1002/bjs.9835. [PubMed: 25963411].

11. Moore WA, Khatri G, Madhuranthakam AJ, Sims RD, Pedrosa I. Added value of diffusion-weighted acquisitions in MRI of the abdomen and pelvis. AJR Am J Roentgenol. 2014;202(5):995-1006. doi: 10.2214/AJR.12.9563. [PubMed: 24758652].

12. Konstantinidis KM, Anastasakou KA, Vorias MN, Sambalis GH, Georgiou MK, Xiarchos AG. A decade of laparoscopic appendectomy: presentation of 1,026 patients with suspected appendicitis treated in a single surgical department. J Laparoendosc Adv Surg Tech A. 2008;18(2):248-58. doi: 10.1089/lap.2006.0209. [PubMed: 18373452].

13. Koh DM, Collins DI. Diffusion-weighted MRI in the body: applications and challenges in oncology. AJR Am J Roentgenol. 2007;188(6):1622-35. doi:10.2214/AJR.06.1403. [PubMed:17515386].
14. Rathod SB, Kumbhar SS, Nanivadekar A, Aman K. Role of diffusionweighted MRI in acute pyelonephritis: a prospective study. Acto Radiol. 2015;56(2):244-9. doi: 10.1177/0284185114520862. [PubMed: 24443116].

15. Stoker J, van Randen A, Lameris W, Boermeester MA. Imaging patients with acute abdominal pain. Radiology. 2009;253(1):31-46. doi 10.1148/radiol.2531090302. [PubMed: 19789254].

16. Bruegel M, Gaa J, Waldt S, Woertler K, Holzapfel K, Kiefer B, et al. Diagnosis of hepatic metastasis: comparison of respirationtriggered diffusion-weighted echo-planar MRI and five t2-weighted turbo spin-echo sequences. AJR Am J Roentgenol. 2008;191(5):1421-9. doi: 10.2214/AJR.07.3279. [PubMed:18941080].

17. Sumi M, Van Cauteren M, Nakamura T. MR microimaging of benign and malignant nodes in the neck. AJR Am J Roentgenol. 2006;186(3):749-57. doi: 10.2214/AJR.04.1832. [PubMed: 16498102].

18. Inci E, Kilickesmez O, Hocaoglu E, Aydin S, Bayramoglu S, Cimilli T. Utility of diffusion-weighted imaging in the diagnosis of acute appendicitis. Eur Radiol. 2011;21(4):768-75. doi: 10.1007/s00330-010-19816. [PubMed: 20924585].

19. Avcu S, Cetin FA, Arslan H, Kemik O, Dulger AC. The value of diffusionweighted imaging and apparent diffusion coefficient quantification in the diagnosis of perforated and nonperforated appendicitis. $D i$ agn Interv Radiol. 2013;19(2):106-10. doi: 10.4261/1305-3825.DIR.607012.1. [PubMed: 23266968].

20. Pinto Leite N, Pereira JM, Cunha R, Pinto P, Sirlin C. CT evaluation of appendicitis and its complications: imaging techniques and key diagnostic findings. AJR Am J Roentgenol. 2005;185(2):406-17. doi: 10.2214/ajr.185.2.01850406. [PubMed: 16037513].

21. Horrow MM, White DS, Horrow JC. Differentiation of perforated from nonperforated appendicitis at CT. Radiology. 2003;227(1):46-51. doi: 10.1148/radiol.2272020223. [PubMed: 12615997].

22. Foley TA, Earnest F, Nathan MA, Hough DM, Schiller HJ, Hoskin TL Differentiation of nonperforated from perforated appendicitis: accuracy of CT diagnosis and relationship of CT findings to length of hospital stay. Radiology. 2005;235(1):89-96. doi:10.1148/radiol.2351040310. [PubMed: 15749978].

23. Ming PC, Yan TY, Tat LH. Risk factors of postoperative infections in adults with complicated appendicitis. Surg Laparosc Endosc Percutan Tech. 2009;19(3):244-8. doi: 10.1097/SLE.0b013e3181a4cda2. [PubMed: 19542855].

24. Hartwich J, Luks FI, Watson-Smith D, Kurkchubasche AG, Muratore CS, Wills HE, et al. Nonoperative treatment of acute appendicitis in children: A feasibility study. J Pediatr Surg. 2016;51(1):111-6. doi: 10.1016/j.jpedsurg.2015.10.024. [PubMed: 26547287].

25. Tanaka Y, Uchida H, Kawashima H, Fujiogi M, Takazawa S, Deie K, et al. Long-term outcomes of operative versus nonoperative treatment for uncomplicated appendicitis. J Pediatr Surg. 2015;50(11):1893-7. doi: 10.1016/j.jpedsurg.2015.07.008. [PubMed: 26259556].

26. Vons C, Barry C, Maitre S, Pautrat K, Leconte M, Costaglioli B, et al Amoxicillin plus clavulanic acid versus appendicectomy for treatment of acute uncomplicated appendicitis: an open-label, noninferiority, randomised controlled trial. Lancet. 2011;377(9777):1573-9. doi: 10.1016/S0140-6736(11)60410-8. [PubMed: 21550483].

27. Bittencourt LK, Matos C, Coutinho AJ. Diffusion-weighted magnetic resonance imaging in the upper abdomen: technical issues and clinical applications. Magn Reson Imaging Clin N Am. 2011;19(1):111-31. doi: 10.1016/j.mric.2010.09.002. [PubMed: 21129638].

28. Kele PG, van der Jagt EJ. Diffusion weighted imaging in the liver. World J Gastroenterol. 2010;16(13):1567-76. doi: 10.3748/wjg.v16.i13.1567. [PubMed: 20355235].

29. Parker MC, Ellis H, Moran BJ, Thompson JN, Wilson MS, Menzies D, et al. Postoperative adhesions: ten-year follow-up of 12,584 patients undergoing lower abdominal surgery. Dis Colon Rectum. 2001;44(6):8229. discussion 829-30. doi: 10.1007/BF02234701. [PubMed: 11391142] 
30. Leung TT, Dixon E, Gill M, Mador BD, Moulton KM, Kaplan GG, et al. Bowel obstruction following appendectomy: what is the true incidence?. Ann Surg. 2009;250(1):51-3. doi: 10.1097/SLA.0b013e3181ad64a7. [PubMed: 19561482].

31. Salminen P, Paajanen H, Rautio T, Nordstrom P, Aarnio M, Rantanen T, et al. Antibiotic Therapy vs Appendectomy for Treatment of Uncomplicated Acute Appendicitis: The APPAC Randomized Clinical Trial.JAMA.2015;313(23):2340-8. doi:10.1001/jama.2015.6154. [PubMed: 26080338].

32. Lane JS, Schmit PJ, Chandler CF, Bennion RS, Thompson JJ. Ileoce- cectomy is definitive treatment for advanced appendicitis. Am Surg. 2001;67(12):1117-22. [PubMed: 11768813].

33. Thompson JJ, Bennion RS, Schmit PJ, Hiyama DT. Cecectomy for complicated appendicitis. J Am Coll Surg. 1994;179(2):135-8. [PubMed: 8044380].

34. Bixby SD, Lucey BC, Soto JA, Theysohn JM, Ozonoff A, Varghese JC. Perforated versus nonperforated acute appendicitis: accuracy of multidetector CT detection. Radiology. 2006;241(3):780-6. doi: 10.1148/radiol.2413051896. [PubMed: 17114626]. 